\title{
Underwater Optical Observation, How to Improve Visibility
}

\author{
Mohcine BOUDHANE, Ojars BALCERS \\ Vidzeme University of Applied Sciences, faculty of Socio-Technical Systems Engineering, \\ Valmiera, Latvia \\ mohcine.boudhane@va.lv, ojars.balcers@va.lv
}

\begin{abstract}
Computer vision is an interdisciplinary field that deals with how computers can be made for gaining high-level understanding from digital images or videos. Fish detection and monitoring is an important topic in computer vision. The growth of high-powered computers, the evolution of high-quality video cameras with low cost and the growing need for automated video analysis have caused more interest in the development of underwater inspection. However, in the sea, the spread of light and sound are not uniform. Therefore, visibility becomes increasingly difficult due to the physical properties of the water. This fact affects negatively of detection accuracy. In this paper, we present method for underwater image enhancement. Dark Channel prior, will be responsible to adjust colour channels in order to get more visibility in images. The algorithm is tested to many images. Experimental results demonstrates a good quality improvement in underwater images.
\end{abstract}

Keywords: Underwater image processing, Optical images, Image enhancement, Image visibility, Underwater technology

\section{Introduction}

Oceans and seas are fragile environments. It contains millions of plant and animal species. Exploration, understanding and investigation of underwater activities is now becoming paramount. Digital image processing is used to solve a wide variety of problems. Although unrelated, these problems usually require methods that can improve information for the interpretation of human visual analysis. Image processing procedures such as image enhancement and restoration are used to process degraded or fuzzy images. 
However, the use of underwater images presents serious problems compared to images in air environment. Generally, underwater visibility is poor, even using very sophisticated equipment (Jaffe, 2015). The major difficulty in processing underwater images comes from the attenuation of light. Legris (Legris et al., 2003) concluded that the attenuation of light limits the visibility distance, about twenty meters in clear water, and less than five meters in turbid water. The damage caused by absorption and that of diffusion changes geographically. Practically, coastal waters are generally more turbid than deep waters because they are more loaded with terrigenous particles. This fact affects negatively to underwater images visibility.

In addition, Outdoor landscapes are usually reduced to some extent by the influence of the medium underwater. Light is refracted, scattered or absorbed through these media. The haze in this medium are generated in this way. When light reaches the camera through these media, it will inevitably be weakened to some extent. The pixel value of each point is affected by its ambient light. These effects result in a decrease in image contrast and color distortion. So we need an image defogging algorithm to restore the original look of the scene. Since most computer vision algorithms are based on the same assumptions of the image and the scene itself, image defogging is crucial. In addition to image restoration, since the thickness of the fog in the foggy image is related to the depth of the scene, it can also be used to estimate the depth of field for further use in photometric, three-dimensional reconstruction and other fields. However, due to the thickness of the fog and the depth of the unknown scene, the difficulty of the problem is also increased. For the case of only one image, image defogging becomes a problem of insufficient constraint. Therefore, predecessors have tried many ways to make up for the missing information. For example, a polarized light filter is used to filter out the dispersion of light in some directions, or to use an image of the same scene at different angles and different weathers, or to use some known 3D models or scene depths to increase unknown information. In recent years, some methods that use prior knowledge of images have made good progress, such as maximizing the contrast of images (He et al., 2011, Boudhane and Balcers, 2019, Pan et al., 2016).

However, the drawback of these methods is that they cannot handle images with large fog, and will fail when their a priori assumptions are not true. The method proposed in (Xie et al., 2010) is based on the statistical information of the fog-free image, and the concept of dark channel is proposed for the defogging of a single image. The prior knowledge found is as follows: For a fog-free image without a sky portion, there is at least one pixel in the R, G, and B channels where the gray value is extremely low to close to zero. For a foggy image, the pixel value with a lower gray value than the entire image is mainly due to the influence of atmospheric light, so it is not as low as close to zero. At the same time, the use of pixels with lower gray scale in the foggy image can be roughly used for the estimation of atmospheric light. Using the prior knowledge of atmospheric light, we can restore the original appearance of the scene.

Therefore, our goal is to exploit the underwater images in order to identify elements that it contains. In this paper, we propose a method of improving the visibility of images 
using the dark channel prior. This method change the colors levels in raw images, and adjust it until we get a clear images. Pre-processing using this method will certainly improve detection accuracy in underwater images. This paper is structured as follows. First, we will describe, theoretically, the proposed enhancement method. Then, we show some experimental results, thus our future work.

\section{Underwater visibility analysis}

In general, visibility corresponds to the possibility of seeing at a certain distance. When diving, visibility beyond 15 meters is considered good. It is bad below 5 meters and excellent above 30 meters (depend to the nature of water). Visibility is a very subjective concept. It depends on the view of the person who determines it, or to the sensor involved to observe in this environment. But it also depends on more specific elements such as the turbidity of the water, the ambient light (which itself depends on the nature of the environment and the sunshine on the surface), the degree of pollution of the water, the hustle and bustle of the sea, the quality of the diving mask.

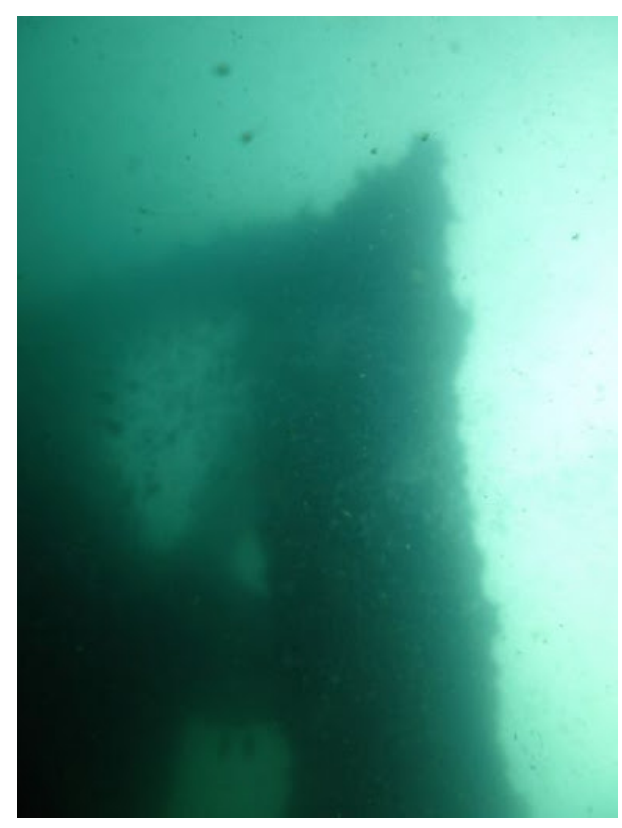

Fig. 1. Examples of images captured in deep sea.

Fig. 1 shows some images captured in the deep water. These images presents high degree of noise that make the identification of its content very difficult. In the other 
hand, from a technical point of view, light crosses the air in a certain direction and propagates in a straight line. Then, when it comes into contact with the transparent object (When it changes medium, or when the medium is no longer homogeneous), it retains a straight line trajectory, but suddenly changes direction. It is then deviated: this is the phenomenon of refraction (fig. 2).

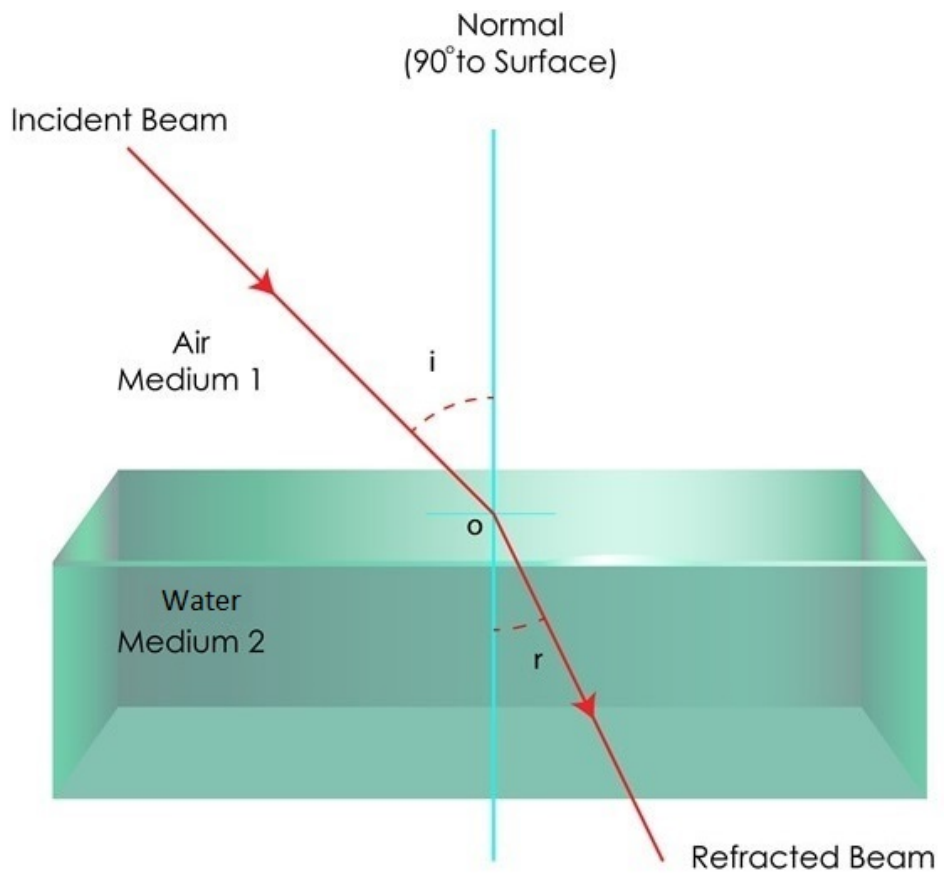

Fig. 2. Reflexion of light propagated from medium 1 to medium 2.

Consequently, the visibility is perturbed. In instance, if a fisherman sees a fish from the surface, does not means that he sees its right position under water. The fish viewed is not really in the same position due to reflexion phenomena. In other words, the fisherman sees only a reflexion of the fish on the surface that does not represents the real position of the fish. Fig. 3 illustrates concretely the reflexion phenomena when a fisherman sees from the surface of water.

Since the visibility is poor, an automated processing of underwater images is mandatory. It would ameliorate the images quality in order to identify the element that contains. This method it could be applied in different field as well as fishery, aquaculture, mines detection, underwater operations, etc. 


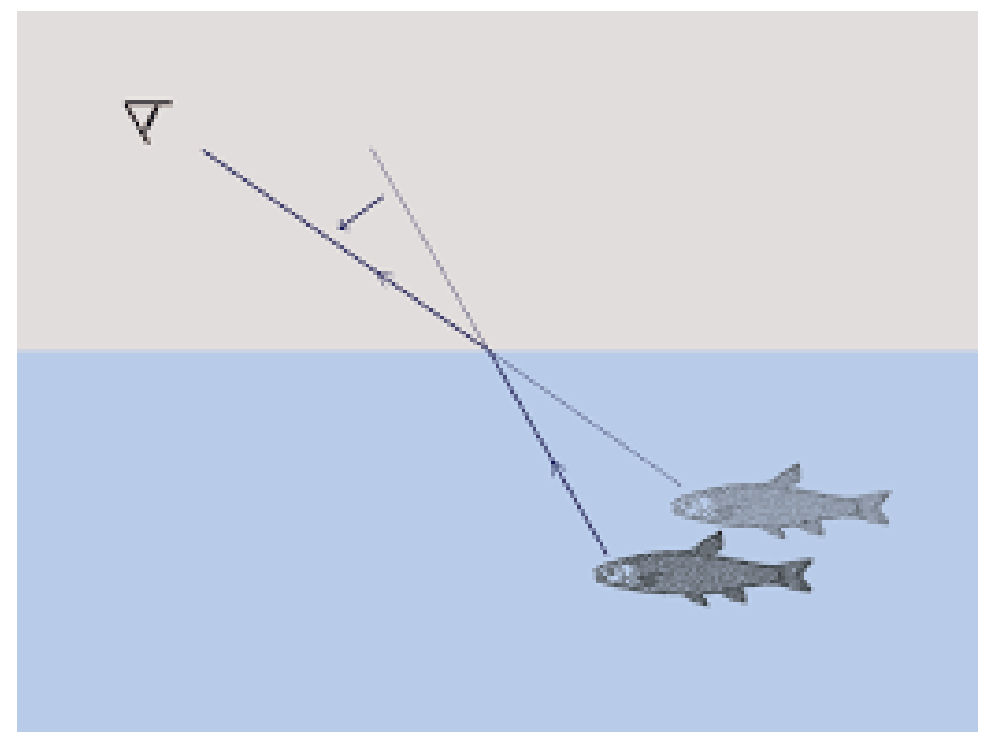

Fig. 3. Reflexion phenomena of water when a fisherman sees from the surface. In bold defines the fish in its real position. But when we look from the surface, the fish is seen in another position different to the real one due to the reflexion phenomena.

\section{Theoretical principles}

In this section, we describe the theoretical principles, where we represent the gray value of the image seen, ie the gray value of the foggy image to be processed. The image is mainly composed of two parts: the first part is the contribution of the scene light itself, corresponding to the first part of the right side of the formula, where $\mathrm{J}$ is the scene image we want to recover, $t$ is the transmission rate of the medium, representing the part of the original scene that reaches the camera. $\mathrm{J}$ describes the degree of direct attenuation of the image. The second part is the contribution of the light penetrated in the medium, A corresponds to atmospheric light, and (1-t) is the transmission rate of atmospheric light representing the part of atmospheric light reaching the camera, describing the influence of the atmosphere in the image.

The goal of the proposed image enhancement is: given I, the value of $t, A$ is required to recover the value of J. So this is a problem of insufficient constraints. At the same time, the transmission rate of the medium and the depth of the scene satisfy the following relationship:

$$
I(x)=J(x) t(x)+(1-t(x)) A
$$

where, $\mathrm{t}$ is the transmission rate, $\mathrm{A}$ is the scattering factor of the atmosphere, and $\mathrm{d}$ is the depth of the scene. After obtaining the transmission rate, we can use this formula to 
find the depth of the scene.

$$
J^{\text {dark }}(x)=\min _{y}\left(\min _{c \in\{r, g, b\}}\left(J^{c}(x)\right)\right)
$$

where is the scattering coefficient of the atmosphere and $d$ is the scene depth. The equation reveals the relationship between scene depth and medium transmission.

\section{Proposed model}

The concept of a dark channel is based on the observation that in a fog-free color image without a sky portion, at least one of the $\mathrm{R}, \mathrm{G}$, and $\mathrm{B}$ channels contains pixels with gray values that are extremely low to close to zero. Similarly, after the image is divided, the minimum value of the gray value corresponding to the block is also close to zero. For each pixel in a color image, calculate the pixel value with the smallest gray value among the three channels corresponding to it, as the pixel value of the point, after processing all the pixels, the corresponding image is obtained. The grayscale image of the lowest gray value. Then, the gray image is patched, and the lowest gray value in each block is used as the gray value of all the pixels of the block. then, the dark channel of the image is obtained.

In addition, the proposed model take on consideration all parameters without prior knowledge of the scene. The model will also reduce the number of equation generated by each color channel. In fact, we use a three dimensional matrix to represent the raw image that includes all color channels R, G, and B. In this model we suppose that the original image is composed by two components: shading 1 and reflectance $\mathrm{R}$.

$$
J(x)=l(x) R(x)
$$

form (1), we obtain:

$$
I(x)=t(x) l(x) R(x)+(1-t(x)) A
$$

We assume that the reflectance $\mathrm{R}$ is constant in the each region.

$$
\mathrm{R}(\mathrm{x})=\mathrm{R}(\text { const })
$$

In order to measure the two unknowns (shading 1 and medium light A) independently, we proceed by multiple derivation using a Piece-wise constant albedo (Biswas et al., 2009). The above calculation process is described by the following formula.

$$
I_{A}(x)=<I(x), A>=t(x) l^{\prime}(x) \eta+(1-t(x))(A)
$$

and

$$
I_{R^{\prime}}(x)=<I(x), R^{\prime}>=t(x) l^{\prime}(x)
$$




\section{Experimental results}

Previous work has addressed the problem of restoring images acquired in underwater scenes from several perspectives (Boudhane et al., 2014, such as the use of special purpose equipment, stereo images in (Halseet al., 2008) (see demonstration in fig. 4), or else the polarization filters as in (Wolff et al., 1995) (fig. 5).

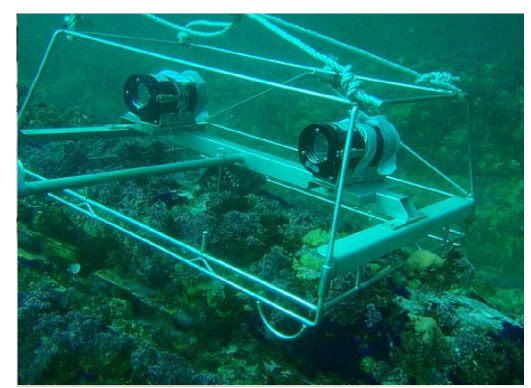

(a)

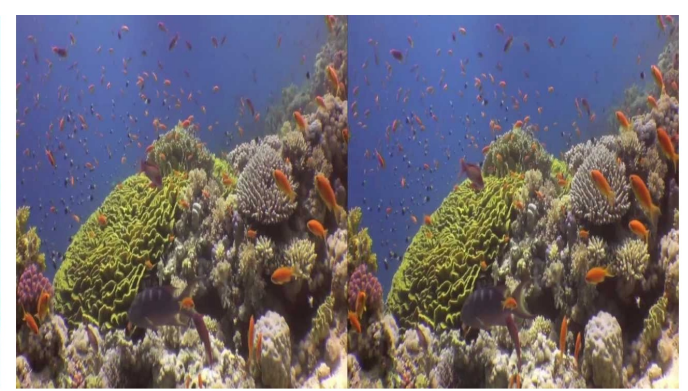

(b)

Fig. 4. Traditional methods stereo imaging. in the left (a): stereo camera system, in the right(b): The view with each cameras, (reference: https ://www.youtube.com/watch?v=j6HiRzsTxDU).

Despite the improvements obtained by these two approaches, they still have several limits. For example, methods that rely on specialized equipment are expensive and complex.

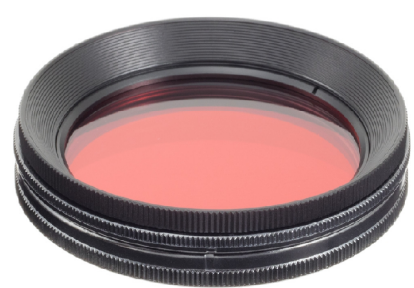

(a)

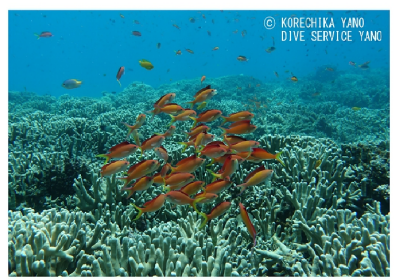

(b)

Fig. 5. Traditional methods stereo imaging. in the left (a): polarizer filter, in the right(b): The view without (in left), and using the polarizer filter (right). source: http://www.inon.jp/products/filter/variableredfilter.html 
The use of polarizers, for example, requires moving parts, and is difficult to implement in automatic acquisition tasks. In an approach of the stereovision system, the problem of correspondence becomes even more difficult because of the harsh effects imposed by the environment. Multiple image methods require at least two images of the same scene taken under different environmental conditions, which makes them unsuitable for real-time applications (Buades et al., 2011). So the problem of restoring images for underwater scenes still requires a lot of research effort, despite the progress that has already been achieved. For this reason comes the proposed method.

Fig.6 shows the comparison of the enhancement effect before and after refinement of the transmission rate. In contrast to the effect, in the unrefined image, the boundary will produce and refine the image. The boundary will produce the same aura as the patch. The refinement is to remove the artificial effect and restore the image to the basic unit. As shown in the figure, the algorithm illustrates a good progress in term of image quality improvement. It has also the ability to enhance the image quality, and edge preservation, and it is successfully reconstructed colors in underwater images. That will certainly improve the detection accuracy.

\section{Conclusion}

In this paper we presented an underwater image denoising algorithm, where we implemented an algorithm based on no statistical hypothesis. This last is based on a reliable physical imaging model using noise (fog) removal.The main objective of our method was to create a denoising algorithm by keeping original information on the scene. The proposed approach is computed on square neighborhoods, this, Block artifacts and halos are reduced by using a soft-matting algorithm. As results we obtained images with high degree of visibility. This method could be integrated in underwater robots sush as AUVs and ROVs. However, when the images are captured more deeper, the color repartition won't be uniform. In future work, we plan to take on consideration these effect by testing our algorithm in different environmental conditions.

\section{Acknowledgment}

This research has been supported by a grant from the European Regional Development Fund project "The Study of Computer Vision Algorithms for Underwater Fish Inspection" No. 1.1.1.2/VIAA/2/18/348 within the Activity 1.1.1.2 "Post-doctoral Research Aid". 

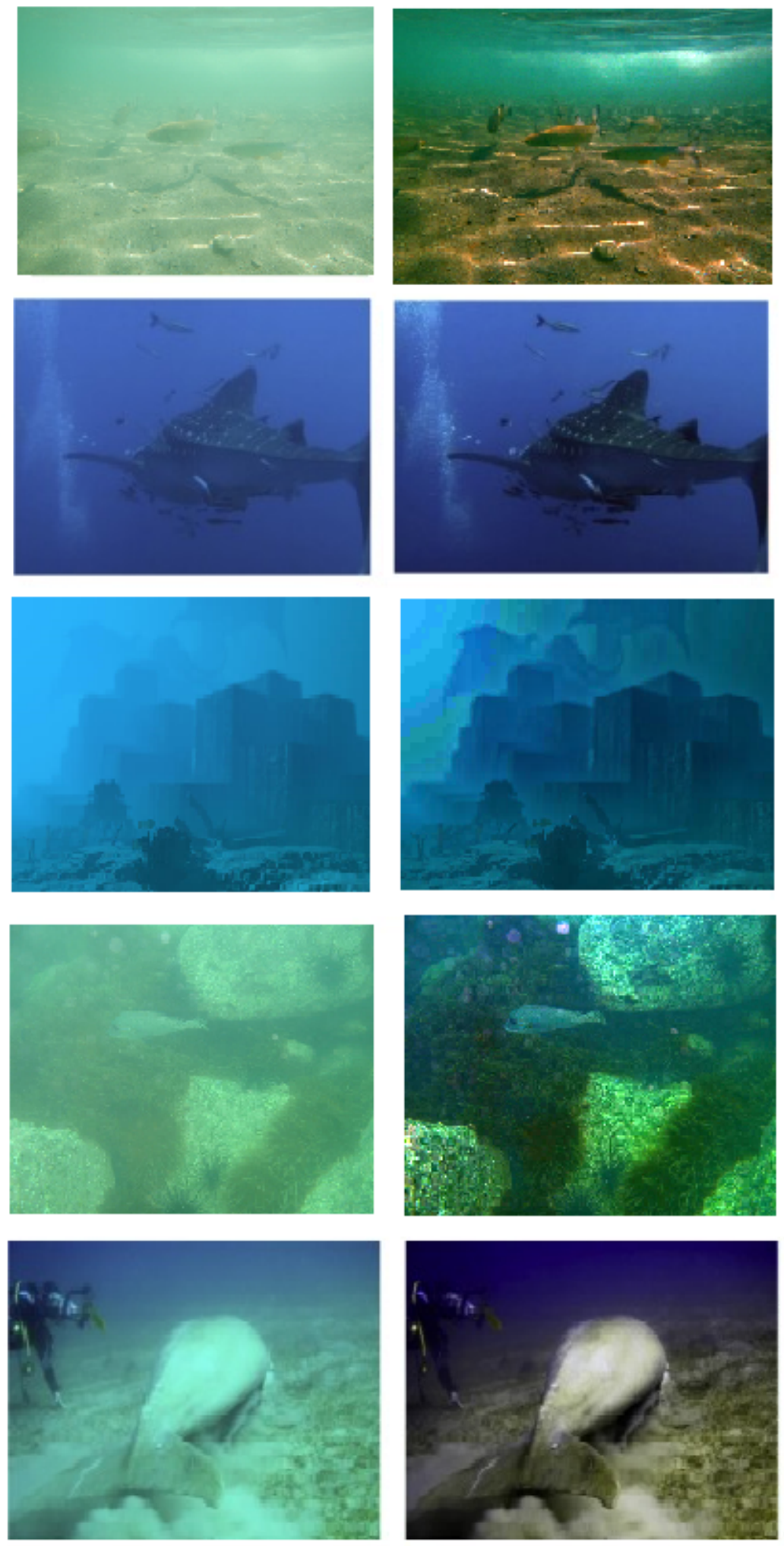

(a)

(b)

Fig. 6. Experimental results. In the left (a): raw images, in the right(b): enhanced images using the proposed approach. 


\section{References}

Biswas, S., Aggarwal, G., Chellappa, R. (2009)., Robust estimation of albedo for illuminationinvariant matching and shape recovery", IEEE Trans Pattern Anal Mach Intell. 2009 May;31(5):884-99. doi: 10.1109/TPAMI.2008.135.

Boudhane M., Badri-Hoeher S., Nsiri B. (2014) "Optical fish estimation and detection in noisy environment" IEEE Oceans - St. John's, 2014. pp1-6.

Boudhane M., Balcers O. (2019) "Underwater Image Enhancement Method Using Color Channel Regularization and Histogram Distribution for Underwater Vehicles AUVs and ROVs", International Journal of Circuits. 13. 571-578.

Buades A., Coll B., Morel JM. (2011) "Non-Local Means Denoising””, IPOL Journal - Image Processing, volume $=$ online, 2011.

Halse S. (2008) "Pioneering stereo-BRUV for long-term monitoring in Africa", Department of Ichthyology and Fisheries Science, Rhodes University.

He K., Sun J., Tang X. (2011) "Single Image Haze Removal Using Dark Channel Prior," in IEEE Transactions on Pattern Analysis and Machine Intelligence, vol. 33, no. 12, pp. 2341-2353, Dec. 2011. doi: 10.1109/TPAMI.2010.168

Jaffe J.S. (2015) "Underwater Optical Imaging: The Past, the Present, and the Prospects”, IEEE Journal of Oceanic Engineering, Volume:40, Issue:3, pp683-700.

Legris M., Lebart K., Fohanno F., Zerr B. (2003) "Les capteurs d'imagerie en robotique sousmarine: tendances actuelles et futures" Saint Martind'Hres,France: GRETSI.

Pan J., Sun D., Pfister H., Yang M. (2016) ”Blind Image Deblurring Using Dark Channel Prior," 2016 IEEE Conference on Computer Vision and Pattern Recognition (CVPR), Las Vegas, NV, 2016, pp. 1628-1636. doi: 10.1109/CVPR.2016.180

Wolff L.B. (1995) "Applications of polarization camera technology", IEEE Expert (Volume : 10, Issue : 5, Oct 1995$)$, pp30-38.

Xie, B., Guo, F., Cai, Z. (2010). Improved Single Image Dehazing Using Dark Channel Prior and Multi-scale Retinex, 2010 International Conference on Intelligent System Design and Engineering Application, DOI: 10.1109/ISDEA.2010.141.

Received March 5, 2020 , accepted March 17, 2020 